
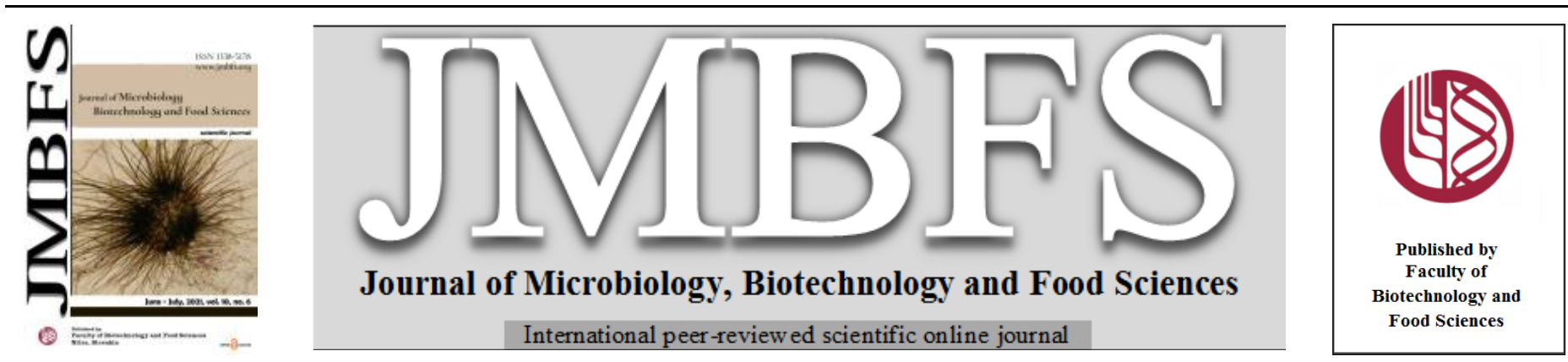

\title{
PREVALENCE AND ANTIBIOTIC RESISTANC PATTERNS OF CAMPYLOBACTER SPECIES ISOLATED FROM DIFFERENT SOURCES IN EYGPT
}

\author{
El-sayed Y. El-Naenaeey ${ }^{1}$, Marwa I. Abd El-Hamid ${ }^{1}$, Eman K. Khalifa $*^{2}$ \\ Address(es): Eman Khaled Khalifa, \\ ${ }^{1}$ Zagazig University, Faculty of Veterinary Medicine, Department of Microbiology, $19^{\text {th }}$ Saleh Abo Rahil Street, El-Nahal, 44511, Zagazig, Sharkia, Egypt. \\ ${ }^{2}$ Zagazig University, Faculty of Veterinary Medicine, Department of Microbiology, Mansoob Street, 44511, Mit-Ghamr, Daqahliyah, Egypt.
}

*Corresponding author: ek41454@gmail.com

https://doi.org/10.15414/jmbfs.3723

\section{ARTICLE INFO}

Received 16. 9. 2020

Revised 19. 1. 2021

Accepted 20. 1. 2021

Published 1. 6. 2021

Regular article

OPEN $\partial$ ACCESS

\begin{abstract}
Campylobacter food poisoning is understated in developing countries. To determine the occurrence and antimicrobial resistance of this pathogen in Zagazig City, Sharkia Governorate, Egypt, a total of 286 samples from chicken (195), cattle (47) and human (44) were collected. Bacteriological examination of the collected samples revealed high prevalence rates of Campylobacter species from human stool, chicken and raw milk samples $(90.91 \%, 86.15 \%$ and $82.98 \%$, respectively). C. jejuni was recognized as the most frequently recovered species (63.29\%). There were no significant effects on the prevalence of Campylobacter spp. based on the sample type ( $p=$ 0.54). Thirty eight campylobacter isolates were confirmed molecularly using PCR amplification of 23S rRNA gene. Furthermore, PCR targeting mapA and ceuE genes were then applied for the confirmation of $C$. jejuni and $C$. coli isolates, respectively. The antibiotic resistance results showed that all isolates (100\%) were resistant to ampicillin, amoxicillin and erythromycin. On the other hand, the lowest resistance rates were detected against amikacin, impenem and cefoxitin (28.74\%, 32.39\% and 47.77, respectively). In total, 207 $(83.81 \%)$ campylobacter isolates were MDR, while 38 isolates (15.38\%) and 2 chicken C. jejuni isolates (0.81\%) were XDR and PDR, respectively. One hundred and forty five antimicrobial resistance profiles were generated with an MAR index of 0.45 or greater. Our results suggested that the presence of MDR campylobacters in chicken and raw milk, specifically to erythromycin and/ or ciprofloxacin aggravates the human health alarm and emphasizes the necessity to educate the consumers about the safeness and the goodness of such foods.
\end{abstract}

Keywords: Campylobacter spp., Prevalence, Multiple drug resistance, mapA, ceuE

\section{INTRODUCTION}

Thermophilic Campylobacter species, mainly Campylobacter jejuni (C. jejuni) and Campylobacter coli (C. coli), have been considered as major causes of acute bacterial gastroenteritis globally. They are microaerophilic microorganisms growing best in an atmosphere containing approximately $10 \% \mathrm{CO}_{2}$ and $5 \% \mathrm{O}_{2}$ at $41.5^{\circ} \mathrm{C}$ (Humphrey et al., 2007). Majority of human illness are attributed to $C$. jejuni $(80-85 \%)$, while the residual cases are credited with $C$. coli (NarvaezBravo et al., 2017).

Campylobacter species colonize the intestine of many farm animals (Silva et al., 2011). The predominant reservoir of these pathogens is chicken and it carries them with no clinical signs (Sahin et al., 2002). This bacterium is transmitted to humans via contaminated undercooked foods of animal origin, especially undercooked chicken meat and unpasteurized dairy products (Gharbi $\boldsymbol{e t}$ al., 2018).

With a low infective dose, Campylobacter species cause a gastrointestinal infection known as campylobacteriosis. Campylobacteriosis is an important zoonotic illness with a universal distribution (Thomrongsuwannakij et al., 2017). In the most recent decades, there was an increase in the number of human infection cases in developing and industrialized countries with 96 million cases of acute gastroenteritis and 21 thousand deaths yearly worldwide (WHO, 2015; Abulreesh et al., 2017). Actually, diarrheal disease has a great importance particularly in developing economies, where children $<2$ years may die when infected with these microorganisms (Saeed $\boldsymbol{e t}$ al., 2015). Campylobacteriosis is characterized by inflammatory diarrhea (Humphrey et al., 2007). In some cases, more severe complications like arthritis, septicemia and Guillain-Barré syndrome (GBS) can also occur when infection caused by either C. jejuni or $C$. coli (Garcia-Sanchez et al., 2018).

Human campylobacteriosis is usually self-limiting. However, immunocompromised patients and young age with severe infections may need antimicrobial medications (Skarp et al., 2016). Erythromycin is generally the first drug of choice, but fluoroquinolones and on a smaller scale tetracycline constitutes other options (Szczepanska et al., 2017).

Campylobacters become more resistant to antimicrobials and many strains developed the multi-drug resistant (MDR) pattern to multiple medicaments (Bouhamed et al., 2018). Multi-drug resistant campylobacter, particularly against quinolones and erythromycin, has increased globally and this has triggered worldwide alarms, as they are the main antibiotics used for the therapy of campylobacteriosis (Zhou et al., 2016). Contaminated foods with MDR campylobacter strains harbor a significant hazard to the public health (Szczepanska et al., 2017).

As the incidence of the campylobacter infections has increased, there is an urgent need to take measures to identify the source of the incriminated bacterium (Eberle and Kiess, 2012). In Egypt, several authors reported relatively high prevalence rate of Campylobacter spp. in human; 27.5\% (Abushahba et al., 2018), 9.37\% (Sainato et al., 2018) and 6\% (Ghoneim et al., 2020). At species level; $C$. jejuni and C. coli were previously recorded with prevalence rates $12.3 \%$ and $2.8 \%$, respectively (El-Tras $\boldsymbol{e t}$ al., 2015). While, thirteen $C$. jejuni isolates (17.33\%) were distinguished from 75 diarrheic persons (GHONEIM et al., 2020).

To the best of our knowledge, the previously conducted studies in Zagazig (Awadallah et al., 2014; Abd El Tawab et al., 2018; El-Hamid et al., 2019) stated limited data about the contribution of chicken and raw milk as potential sources of MDR C. jejuni and C. coli infections in humans. Therefore, the aim of the present investigation was to detect Campylobacter species, particularly $C$. jejuni and C. coli in chicken and raw milk as well as human samples using conventional and molecular tools in addition to identifying the antimicrobial resistance profiles of the recovered isolates against 10 different antimicrobial classes. 


\section{MATERIALS AND METHODS}

\section{Sampling}

The study was conducted from March 2017 to September 2019. A total of 286 samples were collected from various sources; chickens $(n=195)$, cattle $(n=47)$ and human $(n=44)$ in Zagazig City, Sharkia Governorate, Egypt. The samples collected from broiler chickens included cloacal swabs $(n=75)$, breast meat $(n=$ $40)$, caecal parts $(n=40)$ and liver $(n=40)$ were collected from retail outlets, while raw milk samples were collected from retail shops. Moreover, human stoo samples were taken from gastroenteritis patients from clinical laboratories. Samples were collected in a sterile Bolton enrichment broth (Oxoid, UK) and transported in an ice box within $3 \mathrm{~h}$ to the laboratory for bacteriological analysis The animal study was endorsed by the committee of Animal Welfare and Research Ethics, Faculty of Veterinary Medicine, Zagazig University. Regarding the human samples, it was approved by the research ethical committee of Faculty of Medicine, Zagazig University and the work was conducted in compliance with the Ethics of the World Medical Association (Declaration of Helsinki) for studies involving humans. Written informed permissions were taken from patients taking part in the research study after a complete explanation of the aim of the study.

\section{Isolation and identification of thermophilic Campylobacter species}

For isolation of Campylobacter species, the collected samples in Bolton enrichment broth were incubated at $41.5^{\circ} \mathrm{C}$ for $24 \mathrm{~h}$ in the culture vessel with headspace less than $1 \mathrm{~cm}$ and firmly capped lids. After enrichment, a loopful of the broth was streaked onto modified Cefoperazone Charcoal Deoxycholate agar, mCCDA (Oxoid, UK) prepared from Campylocater Blood-Free Selective Agar
Base CM0739 and CCDA Selective Supplement SR155 (Oxoid, UK). The plate's incubation was done at $41.5^{\circ} \mathrm{C}$ in darkness for $48 \mathrm{~h}$ under microaerophilic conditions $\left(5 \% \mathrm{O}_{2}, 10 \% \mathrm{CO}_{2}\right.$ and $85 \% \mathrm{~N}_{2}$ ) using CampyGen sachets (Oxoid, UK) (ISO, 2006). The presumptive identification of isolates as $C$. jejuni and $C$. coli was done by biochemical tests including catalase, oxidase, indoxyl acetate and hippurate hydrolysis and susceptibility to cephalothin and nalidixic acid (30 $\mu \mathrm{g}$ /disc, each) (Quinn et al., 1994).

\section{Molecular confirmation of Campylobacter species}

A conventional PCR was used for the confirmation of biochemically identified campylobacter isolates. Bacterial DNA was extracted from fresh cultures using QIAamp DNA mini kit (Qiagen, USA) in accordance with the manufacturer's instructions. Oligonucleotide primers (Metabion, Germany) that specifically amplify target Campylobacter spp. genes were used for molecular identification (Table 1). The PCR was performed in a total reaction volume of $25 \mu \mathrm{L}$ using Taq DNA Polymerase Kit (Invitrogen, USA) in accordance with the manufacturer's instructions. The used concentration of each forward and reverse primers was 20 pmoL. The thermal profile comprised of an initial denaturation step at $94^{\circ} \mathrm{C}$ for 5 min, followed by 35 cycles of denaturation at $94^{\circ} \mathrm{C}$ for $30 \mathrm{sec}$, annealing at temperatures specific for each gene (Table 1) for $45 \mathrm{sec}$ and extension at $72^{\circ} \mathrm{C}$ for $45 \mathrm{sec}$ and finally an extension step at $72^{\circ} \mathrm{C}$ for $10 \mathrm{~min}$. Reference strains of $C$. jejuni (NCTC11322) and C. coli (NCTC11366) were considered positive controls ( the reference strains were kindly obtained from Biotechnology unit, Reference laboratory for Veterinary Quality Control on Poultry production, Animal Health Research Institute, Dokki, Giza, Egypt). PCR-grade water without template was served as a negative control.

Table 1 Oligonucleotide primers used for PCR amplification of Campylobacter specicc genes with their respective annealing temperatures

\begin{tabular}{|c|c|c|c|c|c|}
\hline Specificity & $\begin{array}{c}\text { Target } \\
\text { gene }\end{array}$ & $\begin{array}{c}\text { Oligonucleotide primer sequence } \\
\left(5^{\prime}-3^{\prime}\right)\end{array}$ & $\begin{array}{l}\text { product } \\
\text { ( bp) }\end{array}$ & $\begin{array}{l}\text { Annealing } \\
\text { temperature }\end{array}$ & Reference \\
\hline \multirow{2}{*}{ Genus Campylobacter } & \multirow{2}{*}{ 23S rRNA } & F' TATACCGGTAAGGAGTGCTGGA & \multirow{2}{*}{650} & \multirow{2}{*}{$55^{\circ} \mathrm{C}$} & \multirow{2}{*}{$\begin{array}{l}\text { Wang et al., } \\
2002\end{array}$} \\
\hline & & R' ATCAATTAACCTTCGAGCACCG & & & \\
\hline Campylobacter coli & ceuE & $\begin{array}{c}\text { F'AAT TGA AAA TTG CTC CAA CTA TG } \\
\text { R' TGA TTT TAT TAT TTG TAG CAG }\end{array}$ & 462 & $58^{\circ} \mathrm{C}$ & \multirow{3}{*}{$\begin{array}{l}\text { Shin and Lee, } \\
2009\end{array}$} \\
\hline \multirow{2}{*}{$\begin{array}{l}\text { Campylobacter } \\
\text { jejuni }\end{array}$} & \multirow{2}{*}{ mapA } & F' CTA TTT TAT TTT TGA GTG CTT GT & \multirow[b]{2}{*}{589} & \multirow[b]{2}{*}{$55^{\circ} \mathrm{C}$} & \\
\hline & & R' GCT TTA TTT GCC ATT TGT TTT ATT & & & \\
\hline
\end{tabular}

\section{Antimicrobial susceptibility testing}

Susceptibility of all campylobacter isolates to 22 antimicrobials representing ten different classes was tested using the following antimicrobial discs: $\beta$-lactams [ampicillin, AM $(10 \mu \mathrm{g})$; amoxicillin, AX $(25 \mu \mathrm{g})$; sulbactam-ampicillin, SAM $(20 \mu \mathrm{g})$; amoxicillin-clavulanic acid, AMC $(30 \mu \mathrm{g})$; cephalothin, KF $(30 \mu \mathrm{g})$; cefoxitin, FOX $(10 \mu \mathrm{g})$; cefepime, FEP $(30 \mu \mathrm{g})$; impenem, IMP $(10 \mu \mathrm{g})$ and azetronam, ATM $(30 \mu \mathrm{g})$ ], aminoglycosides [streptomycin, S $(10 \mu \mathrm{g})$ gentamicin, $\mathrm{CN}(10 \mu \mathrm{g})$ and amikacin, AK $(30 \mu \mathrm{g})$ ], macrolides [erythromycin, E $(15 \mu \mathrm{g})$ and azithromycin, AZM $(15 \mu \mathrm{g})$ ], quinolones [ciprofloxacin, CIP $(5 \mu \mathrm{g})$ and nalidixic acid, NA $(30 \mu \mathrm{g})$ ], sulphonamides [trimethoprim-sulfamethaxole, SXT $(25 \mu \mathrm{g})$ ], phenicols [chloramphenicol, C $(30 \mu \mathrm{g})$ ], polypeptides [colstin, CT $(10 \mu \mathrm{g})$ ], oxazolidones [linezolid, LNZ $(30 \mu \mathrm{g})$ ], lincosamides [clindamycin, DA $(2 \mu \mathrm{g})]$ and tetracyclines [doxycycline DO $(30 \mu \mathrm{g})]$ (Oxoid, UK). This test was conducted following the Kirby-Bauer disk diffusion method (Bauer et al., 1966) Each overnight culture of Campylobacter spp. was suspended in sterile norma saline and adjusted to a turbidity of a $0.5 \mathrm{McFarland}$ standard. Each suspension was inoculated with a sterile swab on the entire surface of Mueller Hinton agar plates (Oxoid, UK) supplemented with 5\% sheep blood. After drying the plates, the antimicrobial discs were aseptically placed on the plates. After incubation at $41.5^{\circ} \mathrm{C}$ for $48 \mathrm{~h}$ under the microaerophilic condition, the inhibition zones were measured and interpreted on the Clinical Laboratory Standards Institute (CLSI) guidelines (CLSI, 2016) and the European Committee on Antimicrobial Susceptibility Testing (EUCAST) (EUCAST, 2017). In the cases when CLSI recommendations were not available for campylobacters, the Enterobacteriaceae CLSI guidelines were tracked (CLSI, 2013). The MDR was identified as acquired resistance of a microorganism to at least one antibiotic in three or more antimicrobial categories, while extensively drug resistant (XDR) was identified as resistance of single bacterium to all antibiotics except two or fewer antimicrobial categories and pan drug resistant (PDR) was identified as resistance of a microorganism to all antibiotics in all antimicrobial categories (Magiorakos et al., 2012).

\section{Multiple antimicrobial resistance indexing}

The multiple antimicrobial resistances (MAR) indexing was used to quantify the multi-resistance of campylobacter isolates, as following: MAR index $=a / b$
Where, "a" represents the number of antimicrobials to which the microorganism was resistant and "b" represents the total number of antimicrobials to which the microorganism was tested (Krumperman, 1983).

\section{Statistical analysis}

The results were analyzed using the SPSS v18.0 software (SPSS Inc., Chicago, IL, USA). The occurrence of Campylobacter spp. in different sources and variations in antimicrobial susceptibility between $C$. jejuni and $C$. coli were evaluated using chi square test. Fisher's exact test was applied for smaller number of samples. To visualize the clustering pattern among campylobacter isolates from various hosts, non-metric multidimensional scaling was generated based on sorensen distance using the PC-ORD software.

\section{RESULTS}

\section{Prevalence of thermophilic Campylobacter spp. in the examined samples}

According to the phenotypic identification, Campylobacter spp. were isolated from 247 out of 286 of the examined samples $(86.36 \%) ; 90.91 \%$ from human stool, $86.15 \%$ from chicken samples and $82.98 \%$ from raw milk (Table 2). Of note, each investigated sample contained only one identified campylobacter isolate. The results demonstrated a high isolation rate of chicken Campylobacter spp. from cloacal swabs $(93.33 \%)$, followed by caecal parts $(90 \%)$, chicken liver $(80 \%)$ and breast meat $(75 \%)$. Identification of campylobacters to the species level showed that $C$. jejuni was found to be the predominant species with an overall prevalence rate of $63.29 \%$, while that of $C$. coli was $23.08 \%$. The highest isolation rate of $C$. jejuni was detected in human stool $(72.73 \%)$, followed by raw milk (70.21\%). Meanwhile, the highest isolation rate of $C$. coli was observed in chicken $(26.67 \%)$ (Table 2). Statistically, there were no significant effects on the prevalence of Campylobacter spp. based on the sample type $(p=0.54)$ and on the prevalence of both $C$. jejuni and $C$. coli from different sample types $(p=0.08)$. 
Table 2 Prevalence of thermotolerant Campylobacter spp. in the collected samples

\begin{tabular}{|c|c|c|c|}
\hline \multirow{2}{*}{$\begin{array}{l}\text { Sample type } \\
\text { (No.) }\end{array}$} & \multirow{2}{*}{$\begin{array}{c}\text { Total No. of } \\
\text { Campylobacter spp. } \\
(\%)^{*}\end{array}$} & \multicolumn{2}{|c|}{$\begin{array}{c}\text { No. of Campylobacter spp. } \\
(\%)^{*}\end{array}$} \\
\hline & & C. jejuni & C. coli \\
\hline $\begin{array}{l}\text { Chicken } \\
\text { samples (195) }\end{array}$ & $168(86.15)$ & $116(59.49)$ & $52(26.67)$ \\
\hline $\begin{array}{l}\text { Clocal swabs } \\
(75)\end{array}$ & $70(93.33)$ & $50(66.67)$ & $20(26.67)$ \\
\hline $\begin{array}{l}\text { Breast meat } \\
(40)\end{array}$ & $30(75)$ & $22(55)$ & $8(20)$ \\
\hline Cecal parts (40) & $36(90)$ & $24(60)$ & $12(30)$ \\
\hline $\begin{array}{l}\text { Chicken liver } \\
(40)\end{array}$ & $32(80)$ & $20(50)$ & $12(30)$ \\
\hline Raw milk (47) & $39(82.98)$ & $33(70.21)$ & $6(12.77)$ \\
\hline $\begin{array}{l}\text { Human stool } \\
\text { (44) }\end{array}$ & $40(90.91)$ & $32(72.73)$ & $8(18.18)$ \\
\hline Total (286) & 247 (86.36) & $181(63.29)$ & $66(23.08)$ \\
\hline
\end{tabular}

\section{Molecular confirmation of campylobacter isolates}

Thirty eight campylobacter isolates (29 and 9 biochemically suspected $C$. jejun and $C$. coli isolates, respectively) were confirmed by PCR amplification of $23 \mathrm{~S}$ rRNA gene. All the isolates generated an amplicon of 650 bp size (figure 1). The results showed that all 29 C. jejuni isolates from chicken $(n=19)$, cattle $(n=5)$ and human $(\mathrm{n}=5)$ and $9 C$. coli isolates from chicken $(\mathrm{n}=7)$, cattle $(\mathrm{n}=1)$ and human $(\mathrm{n}=1)$ those were identified phenotypically were confirmed by PCR ampilifications of mapA and ceuE genes with amplicons' sizes of 589 bp (figure 2A) and 462 bp (figure 2B), respectively.

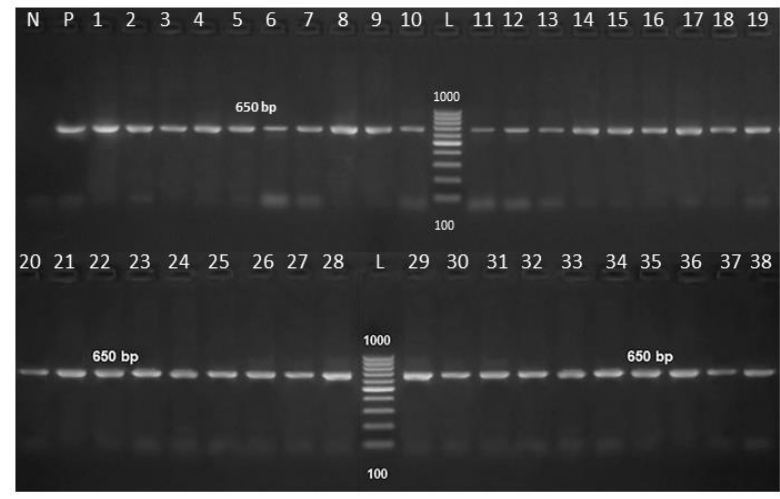

Figure 1 PCR amplification products of 23S rRNA gene of Campylobacter spp (650 bp). Lane L: 100 bp DNA ladder "Marker", lanes 1-38: positive campylobacter isolates from poultry (lanes 1-26), cattle (lanes 27-32) and human (lanes 33-38) origins, lane P: positive control, lane N: negative control.

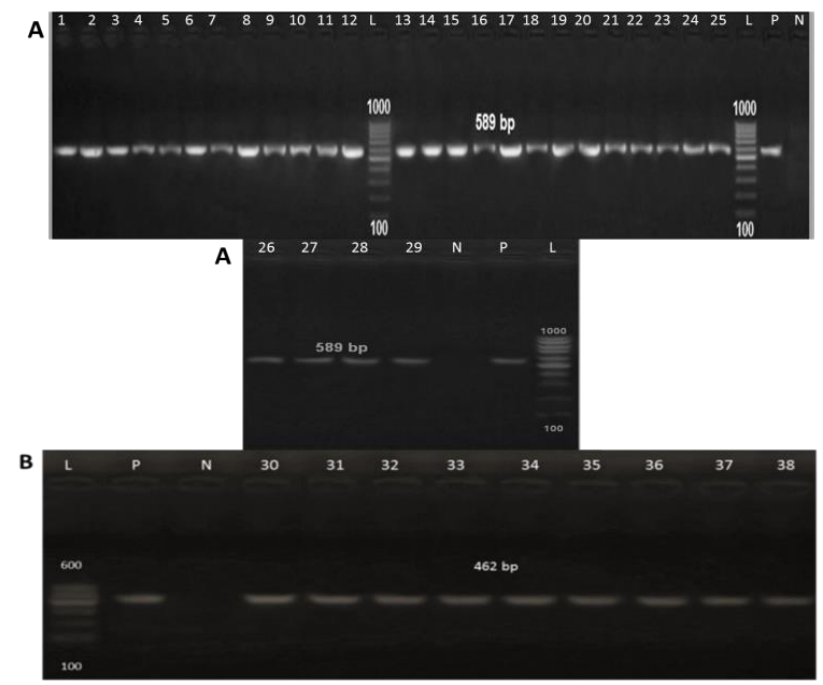

Figure 2 PCR amplification products of mapA gene specific for $C$. jejuni (589 bp) (A) and ceuE gene specific for C. coli (462 bp) (B). Lanes 1-19: C. jejuni from poultry, lanes 20-24: C. jejuni from cattle, lanes 25-29: C. jejuni from human, lanes 30-36: $C$. coli from poultry, lane 37: $C$. coli from cattle, lane 38: $C$. coli from human, lane L: 100 bp DNA ladder "Marker", lane P: positive control, lane N: negative control

Antimicrobial susceptibility profile of the recovered Campylobacter spp. from different sources

Analysis of the antimicrobial resistance of 247 campylobacter isolates against the 22 tested antimicrobial agents demonstrated that all isolates (100\%) were resistant to ampicillin, amoxicillin and erythromycin. Moreover, high resistance rates were observed against trimethoprim-sulfamethaxole $(98.79 \%)$, followed by clindamycin (97.17\%), cephalothin $(96.76 \%)$, azithromycin $(91.09 \%)$ and nalidixic acid $(90.28 \%)$. Additionally, majority of campylobacter isolates were resistant to azetronam $(81.78 \%)$, doxycycline $(81.38 \%)$, amoxicillin-clavulanic acid $(80.97 \%)$, cefepime $(80.57 \%)$, chloramphenicol $(80.16 \%)$, colistin $(75.71 \%)$ and linezolid $(73.28 \%)$. On the other hand, our results showed that amikacin, impenem and cefoxitin had the lowest resistance rates against the tested isolates (28.74\%, 32.39\% and $47.77 \%$, respectively) (Table 3).

Based on Campylobacter species and regardless of the type of the collected samples, our results presented higher resistance rates in $C$. jejuni isolates than $C$. coli for the investigated antibiotics except for cephalothin, cefoxitin, impenem, streptomycin, gentamycin, ciprofloxacin, linezolid, colistin and doxycycline (Figure 3). Statistically, there was a significant effect $(P<0.05)$ on the resistance prevalence between $C$. jejuni and $C$. coli isolates against amoxicillin-clavulanic acid $(84.53 \%$ and $71.21 \%$ ), cefepime $(83.98 \%$ and $71.21 \%)$, azithromycin (93.37\% and $84.85 \%)$, chloramphenicol $(83.43 \%$ and $71.21 \%)$ and colistin (72.38\% and $84.85 \%$ ), respectively. The resistance prevalence in C. jejuni and $C$. coli isolates was significantly higher for 3 of the tested antimicrobials; azetronam $(86.19 \%$ and $69.69 \%)$ and streptomycin $(58.01 \%$ and $77.27 \%)$, respectively $(P<$ $0.01)$ and clindamycin $(100 \%$ and $89.39 \%)$, respectively $(P<0.001)$. Meanwhile, there were no significant differences $(P>0.05)$ in the resistance of $C$. jejuni and $C$. coli isolates against the other antimicrobials; sulbactam-ampicillin, cephalothin, cefoxitin, impenem, gentamycin, amikacin, ciprofloxacin, nalidixic acid, trimethoprim-sulfamethaxole, linezolid and doxycycline $(P$ values $=0.192$, $0.911,0.48,0.27,0.81,0.99,0.54,0.78, .795,0.59$ and 0.22 , respectively) (Supplementary table 1).

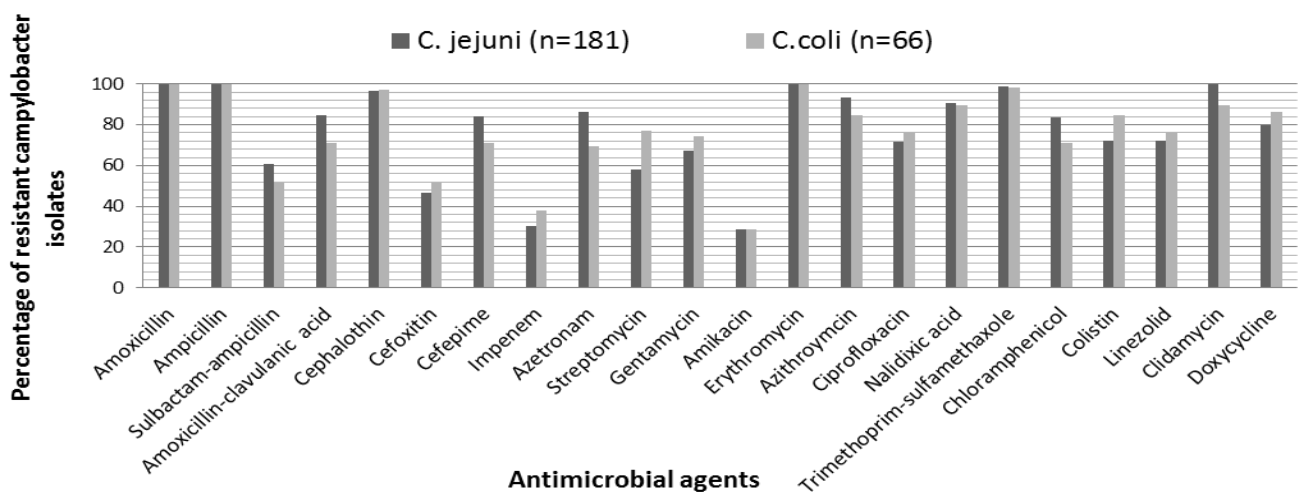

Figure 3 Prevalence of Campylobacter spp. resistance against 22 antimicrobial agents 
According to the isolates' origins, the resistance levels of campylobacter isolates recovered from chicken, cattle and human samples were different (Figure 4). Statistically, there was a significant effect $(P<0.05)$ on the resistance rates between chicken, cattle and human isolates against impenem $(36.9 \%, 15.38 \%$ and $30 \%)$ and nalidixic acid $(94.05 \%, 84.62 \%$ and $80 \%)$, respectively. The difference in rates among chicken, cattle and human isolates were significantly higher for 11 of the tested antimicrobials; cefepime $(78.57 \%, 69.23 \%$ and $100 \%)$, streptomycin $(69.05 \%, 38.46 \%$ and $62.5 \%)$, trimethoprim-sulfamethaxole $(100 \%$, $100 \%$ and $92.5 \%)$ and clindamycin $(98.81 \%, 100 \%$ and $87.5 \%)$, respectively $(P<$ $0.01)$ and sulbactam-ampicillin $(52.38 \%, 92.31 \%$ and $50 \%)$, amoxicillinclavulanic acid $(88.09 \%, 61.54 \%$ and $70 \%)$, azetronam $(81.55 \%, 100 \%$ and
$65 \%)$, gentamycin $(76.19 \%, 35.89 \%$ and $72.5 \%)$, azithromycin $(86.9 \%, 100 \%$ and $100 \%)$, chloramphenicol $(75 \%, 100 \%$ and $82.5 \%)$ and linezolid $(77.98 \%$, $46.15 \%$ and $80 \%)$, respectively $(P<0.001)$. However, there is no significan effect $(P>0.05)$ on the resistance prevalence between campylobacter isolates from the three sources against the other antibiotics; cephalothin, cefoxitin, amikacin, ciprofloxacin, colistin and doxycycline $(P=0.12,0.24,0.9,0.22,0.31$ and, 0.05 , respectively) (Supplementary table 2 ). The clustering pattern of Campylobacter isolates were classified by their species and host. The distance among isolates is of the Sorensen type (Figure 5).

Table 3 Frequency of resistance of thermotolerant Campylobacter spp. recovered from different sources

\begin{tabular}{|c|c|c|c|c|c|c|c|c|}
\hline \multirow{3}{*}{ Antimicrobial group } & \multirow{3}{*}{ Antimicrobial } & \multicolumn{6}{|c|}{ No. of resistant Campylobacter spp. from different sources (\%) } & \multirow{3}{*}{$\begin{array}{l}\text { Total } \\
(247)\end{array}$} \\
\hline & & \multicolumn{2}{|c|}{ Chicken (168) } & \multicolumn{2}{|c|}{ Cattle (39) } & \multicolumn{2}{|c|}{ Human (40) } & \\
\hline & & $\begin{array}{l}\text { C. jejuni } \\
(\mathrm{n}=116)\end{array}$ & $\begin{array}{l}\text { C. coli } \\
(\mathrm{n}=52)\end{array}$ & $\begin{array}{c}\text { C. jejuni } \\
(\mathrm{n}=33)\end{array}$ & $\begin{array}{c}\text { C. coli } \\
(\mathrm{n}=6)\end{array}$ & $\begin{array}{c}\text { C. jejuni } \\
(\mathrm{n}=32)\end{array}$ & $\begin{array}{l}\text { C. coli } \\
(\mathrm{n}=8)\end{array}$ & \\
\hline \multirow{9}{*}{ Beta- lactams } & Amoxicillin & $\begin{array}{c}116 \\
(100) \\
\end{array}$ & $\begin{array}{c}52 \\
(100) \\
\end{array}$ & $\begin{array}{c}33 \\
(100) \\
\end{array}$ & $\begin{array}{c}6 \\
(100) \\
\end{array}$ & $\begin{array}{c}32 \\
(100)\end{array}$ & $\begin{array}{c}8 \\
(100) \\
\end{array}$ & $\begin{array}{c}247 \\
(100)\end{array}$ \\
\hline & Ampicillin & $\begin{array}{c}116 \\
(100) \\
\end{array}$ & $\begin{array}{c}52 \\
(100) \\
\end{array}$ & $\begin{array}{c}33 \\
(100) \\
\end{array}$ & $\begin{array}{c}6 \\
(100) \\
\end{array}$ & $\begin{array}{c}32 \\
(100)\end{array}$ & $\begin{array}{c}8 \\
(100) \\
\end{array}$ & $\begin{array}{c}247 \\
(100) \\
\end{array}$ \\
\hline & Sulbactam-ampicillin & $\begin{array}{c}64 \\
(55.17)\end{array}$ & $24(46.15)$ & $\begin{array}{c}31 \\
(93.94) \\
\end{array}$ & $5(83.33)$ & $\begin{array}{c}15 \\
(46.88) \\
\end{array}$ & $\begin{array}{c}5 \\
(62.5)\end{array}$ & $144(58.29)$ \\
\hline & Amoxicillin-clavulanic acid & $\begin{array}{c}112 \\
(96.55) \\
\end{array}$ & $36(69.23)$ & $\begin{array}{c}20 \\
(60.61)\end{array}$ & $\begin{array}{c}4 \\
(66.67) \\
\end{array}$ & $\begin{array}{c}21 \\
(65.63)\end{array}$ & $\begin{array}{c}7 \\
(87.5) \\
\end{array}$ & $200(80.97)$ \\
\hline & Cephalothin & $\begin{array}{c}111 \\
(95.69)\end{array}$ & $\begin{array}{c}52 \\
(100) \\
\end{array}$ & $\begin{array}{c}32 \\
(96.97) \\
\end{array}$ & $4(66.67)$ & $\begin{array}{c}32 \\
(100)\end{array}$ & $\begin{array}{c}8 \\
(100) \\
\end{array}$ & $239(96.76)$ \\
\hline & Cefoxitin & $\begin{array}{c}54 \\
(46.55) \\
\end{array}$ & $22(42.31)$ & $\begin{array}{c}14 \\
(42.42) \\
\end{array}$ & $\begin{array}{c}4 \\
(66.67) \\
\end{array}$ & $\begin{array}{c}16 \\
(50) \\
\end{array}$ & $\begin{array}{c}8 \\
(100) \\
\end{array}$ & $118(47.77)$ \\
\hline & Cefepime & $\begin{array}{c}98 \\
(84.48) \\
\end{array}$ & $\begin{array}{c}34 \\
(65.38) \\
\end{array}$ & $\begin{array}{c}22 \\
(66.67) \\
\end{array}$ & $\begin{array}{c}5 \\
(83.33) \\
\end{array}$ & $\begin{array}{c}32 \\
(100) \\
\end{array}$ & $\begin{array}{c}8 \\
(100) \\
\end{array}$ & $199(80.57)$ \\
\hline & Impenem & $\begin{array}{c}41 \\
(35.34) \\
\end{array}$ & $\begin{array}{c}21 \\
(40.38) \\
\end{array}$ & $\begin{array}{c}5 \\
(15.15) \\
\end{array}$ & $\begin{array}{c}1 \\
(16.67) \\
\end{array}$ & $\begin{array}{c}9 \\
(28.13) \\
\end{array}$ & $\begin{array}{c}3 \\
(37.5) \\
\end{array}$ & $\begin{array}{c}80 \\
(32.39) \\
\end{array}$ \\
\hline & Azetronam & $\begin{array}{c}101 \\
(87.07) \\
\end{array}$ & $36(69.23)$ & $\begin{array}{c}33 \\
(100) \\
\end{array}$ & $\begin{array}{c}6 \\
(100) \\
\end{array}$ & $\begin{array}{c}22 \\
(68.75) \\
\end{array}$ & $\begin{array}{c}4 \\
(50) \\
\end{array}$ & $202(81.78)$ \\
\hline \multirow{3}{*}{ Aminoglycosides } & Streptomycin & $\begin{array}{c}76 \\
(65.52) \\
\end{array}$ & $\begin{array}{c}40 \\
(76.92) \\
\end{array}$ & $\begin{array}{c}12 \\
(36.36) \\
\end{array}$ & $\begin{array}{c}3 \\
(50) \\
\end{array}$ & $\begin{array}{c}17 \\
(53.13) \\
\end{array}$ & $\begin{array}{c}8 \\
(100) \\
\end{array}$ & $156(63.16)$ \\
\hline & Gentamycin & $\begin{array}{c}90 \\
(77.59) \\
\end{array}$ & $38(73.08)$ & $\begin{array}{c}11 \\
(33.33) \\
\end{array}$ & $\begin{array}{c}3 \\
(50) \\
\end{array}$ & $\begin{array}{c}21 \\
(65.63) \\
\end{array}$ & $\begin{array}{c}8 \\
(100) \\
\end{array}$ & $171(69.23)$ \\
\hline & Amikacin & $\begin{array}{c}30 \\
(25.86)\end{array}$ & $12(23.08)$ & $\begin{array}{c}9 \\
(27.27)\end{array}$ & $\begin{array}{c}3 \\
(50)\end{array}$ & $\begin{array}{c}13 \\
(40.63)\end{array}$ & $\begin{array}{c}4 \\
(50)\end{array}$ & $\begin{array}{c}71 \\
(28.74)\end{array}$ \\
\hline \multirow{2}{*}{ Macrolides } & Erythromycin & $\begin{array}{c}116 \\
(100) \\
\end{array}$ & $\begin{array}{c}52 \\
(100) \\
\end{array}$ & $\begin{array}{c}33 \\
(100) \\
\end{array}$ & $\begin{array}{c}6 \\
(100) \\
\end{array}$ & $\begin{array}{c}32 \\
(100) \\
\end{array}$ & $\begin{array}{c}8 \\
(100) \\
\end{array}$ & $\begin{array}{c}247 \\
(100) \\
\end{array}$ \\
\hline & Azithroymcin & $\begin{array}{c}104 \\
(89.66) \\
\end{array}$ & $42(80.77)$ & $\begin{array}{c}33 \\
(100) \\
\end{array}$ & $\begin{array}{c}6 \\
(100) \\
\end{array}$ & $\begin{array}{c}32 \\
(100) \\
\end{array}$ & $\begin{array}{c}8 \\
(100) \\
\end{array}$ & $225(91.09)$ \\
\hline \multirow{2}{*}{ Quinolones } & Ciprofloxacin & $\begin{array}{c}85 \\
(73.28) \\
\end{array}$ & $41(78.85)$ & $\begin{array}{c}20 \\
(60.61) \\
\end{array}$ & $\begin{array}{c}4 \\
(66.67) \\
\end{array}$ & $\begin{array}{c}25 \\
(78.13) \\
\end{array}$ & $\begin{array}{c}5 \\
(62.5) \\
\end{array}$ & $180(72.87)$ \\
\hline & Nalidixic acid & $\begin{array}{c}110 \\
(94.83) \\
\end{array}$ & $48(92.31)$ & $\begin{array}{c}30 \\
(90.91) \\
\end{array}$ & $\begin{array}{c}3 \\
(50) \\
\end{array}$ & $\begin{array}{c}24 \\
(75) \\
\end{array}$ & $\begin{array}{c}8 \\
(100) \\
\end{array}$ & $223(90.28)$ \\
\hline Sulphonamides & $\begin{array}{l}\text { Trimethoprim- } \\
\text { sulfamethaxole }\end{array}$ & $\begin{array}{c}116 \\
(100) \\
\end{array}$ & $\begin{array}{c}52 \\
(100) \\
\end{array}$ & $\begin{array}{c}33 \\
(100) \\
\end{array}$ & $\begin{array}{c}6 \\
(100) \\
\end{array}$ & $\begin{array}{c}30 \\
(93.75) \\
\end{array}$ & $\begin{array}{c}7 \\
(87.5) \\
\end{array}$ & $244(98.79)$ \\
\hline Phenicols & Chloramphenicol & $\begin{array}{c}90 \\
(77.59) \\
\end{array}$ & $36(69.23)$ & $\begin{array}{c}33 \\
(100) \\
\end{array}$ & $\begin{array}{c}6 \\
(100) \\
\end{array}$ & $\begin{array}{c}28 \\
(87.5) \\
\end{array}$ & $\begin{array}{c}5 \\
(62.5) \\
\end{array}$ & $198(80.16)$ \\
\hline Polypeptides & Colistin & $\begin{array}{c}87 \\
(75) \\
\end{array}$ & $45(86.54)$ & $\begin{array}{c}24 \\
(72.73) \\
\end{array}$ & $\begin{array}{c}3 \\
(50) \\
\end{array}$ & $\begin{array}{c}20 \\
(62.5) \\
\end{array}$ & $\begin{array}{c}8 \\
(100) \\
\end{array}$ & $187(75.71)$ \\
\hline Oxazolidone & Linezolid & $\begin{array}{c}90 \\
(77.59) \\
\end{array}$ & $41(78.85)$ & $\begin{array}{c}15 \\
(45.45) \\
\end{array}$ & $\begin{array}{c}3 \\
(50) \\
\end{array}$ & $\begin{array}{c}26 \\
(81.25) \\
\end{array}$ & $\begin{array}{c}6 \\
(75) \\
\end{array}$ & $181(73.28)$ \\
\hline Lincosamide & Clindamycin & $\begin{array}{c}116 \\
(100) \\
\end{array}$ & $50(96.15)$ & $\begin{array}{c}33 \\
(100) \\
\end{array}$ & $\begin{array}{c}6 \\
(100) \\
\end{array}$ & $\begin{array}{c}32 \\
(100) \\
\end{array}$ & $\begin{array}{c}3 \\
(37.5) \\
\end{array}$ & $240(97.17)$ \\
\hline Tetracyclines & Doxycycline & $\begin{array}{c}90 \\
(77.59)\end{array}$ & $48(92.31)$ & $\begin{array}{c}22 \\
(66.67)\end{array}$ & $\begin{array}{c}5 \\
(83.33) \\
\end{array}$ & $\begin{array}{c}32 \\
(100)\end{array}$ & $\begin{array}{c}4 \\
(50)\end{array}$ & $201(81.38)$ \\
\hline
\end{tabular}

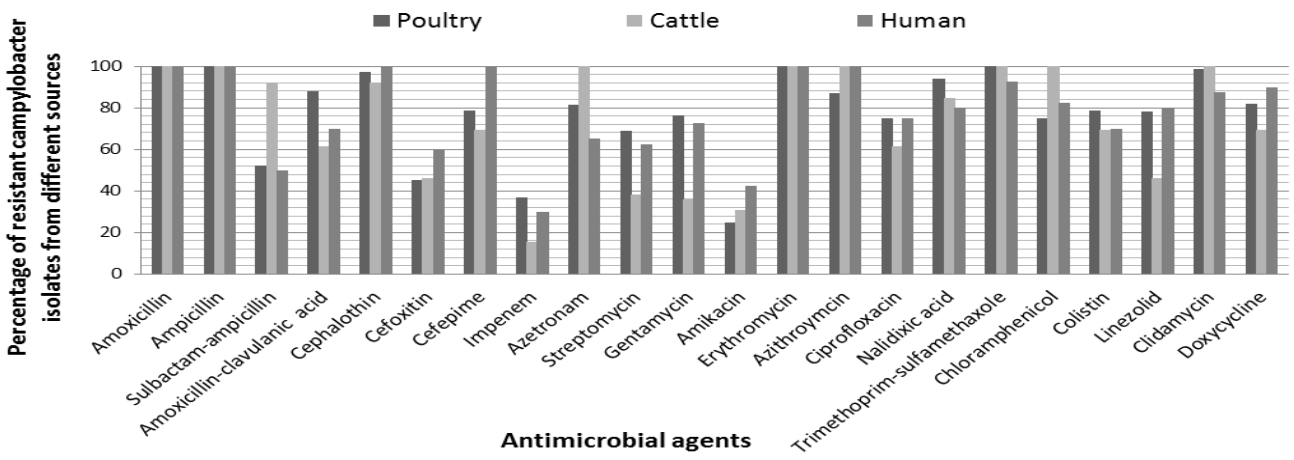

Figure 4 Prevalence of resistance in campylobacter isolates from different sources against 22 antimicrobial agents 


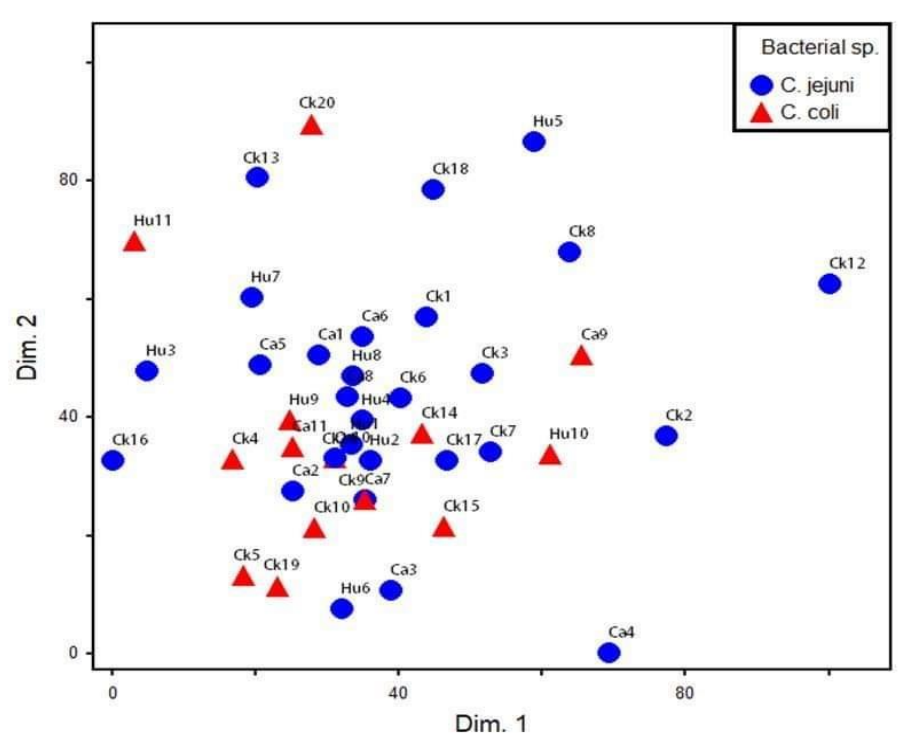

Figure 5 Non-metric multidimensional scaling plot showing the clustering pattern of Campylobacter isolates classified by their species (color and shape of symbol) and host (text above each dot). Each dot shows one isolate $(\mathrm{n}=40)$. (Ck: chicken, Ca: cattle, Hu: human)

Antimicrobial resistance profiles and multiple antimicrobial resistance indices of campylobacter isolates

The obtainable results showed that in chicken isolates, $C$. jejuni isolates were resistant to $7(10.35 \%), 8(21.55 \%), 9(35.34 \%)$ and 10 classes $(32.76 \%)$ of the tested antimicrobial agents while, $C$. coli isolates were resistant to 7 (5.77\%), 8 $(19.23 \%), 9(30.77 \%)$ and 10 classes $(44.23 \%)$ of the investigated antimicrobia agents. Besides, in cattle isolates, $C$. jejuni isolates were resistant to 7 (18.18\%), $8(30.30 \%), 9(33.33 \%)$ and 10 classes $(18.18 \%)$ and in $C$. coli isolates were resistant to $7(16.67 \%), 8$ and $9(33.33 \%$, each) and 10 classes $(16.67 \%)$ of the tested antimicrobial agents. Additionally, in human isolates, C. jejuni isolates were resistant to $8(28.13 \%), 9(40.63 \%)$ and 10 classes $(31.25 \%)$ of the antimicrobial agents and in $C$. coli isolates were resistant to $7(50 \%), 9(37.5 \%)$ and 10 classes $(12.5 \%)$ of the antimicrobial agents (Table 4).

In total, the prevalence of resistance to seven and eight antimicrobial classes was higher in cattle campylobacter isolates $(17.95 \%$ and $30.77 \%$, respectively) than chicken and human campylobacter ones. Meanwhile, the resistance to nine antimicrobial classes was the highest in human isolates (40\%) and that to ten classes was the highest in chicken isolates $(36.31 \%$ ) (Table 4).

Interestingly, it was noticed that $207(83.81 \%)$ isolates of campylobacter were MDR (resistant to 3-8 antimicrobial classes). Additionally, our results identified 38 XDR isolates $(15.38 \%) ; 10(25 \%), 26(15.5 \%)$ and $2(5.1 \%)$ were recovered from human, chicken and cattle samples, respectively. It is important to note the presence of $2(0.81 \%)$ chicken $C$. jejuni those were PDR (Table 4).

Estimating the MAR indices for campylobacter isolates from different sources revealed that all tested chicken isolates presented an MAR index of 0.45 or greater. Moreover, human isolates had an index of 0.59 or greater and cattle isolates revealed an index of 0.63 or greater indicating a high risk source of contamination, where the antibiotics were often used.

Table 4 Multiple antimicrobial resistance index (MAR) of thermotolerant Campylobacter spp. recovered from different sources

\begin{tabular}{|c|c|c|c|c|c|c|c|c|c|c|}
\hline \multirow{3}{*}{ MAR index } & \multirow{3}{*}{$\begin{array}{c}\text { No. of } \\
\text { antimicrobials to } \\
\text { which the isolates } \\
\text { were resistant (22) }\end{array}$} & \multirow{3}{*}{$\begin{array}{l}\text { No. of } \\
\text { AMC }\end{array}$} & \multicolumn{6}{|c|}{ No. of resistant Campylobacter spp. from different sources (\%) } & \multirow{3}{*}{$\begin{array}{l}\text { Total } \\
(247)\end{array}$} & \multirow{3}{*}{$\begin{array}{l}\text { Character } \\
\text { of resistant } \\
\text { strains }\end{array}$} \\
\hline & & & \multicolumn{2}{|c|}{ Chicken } & \multicolumn{2}{|c|}{ Cattle } & \multicolumn{2}{|c|}{ Human } & & \\
\hline & & & $\begin{array}{c}\text { C. jejuni } \\
\text { (116) }\end{array}$ & $\begin{array}{l}\text { C. coli } \\
\text { (52) }\end{array}$ & $\begin{array}{l}\text { C. jejuni } \\
\text { (33) }\end{array}$ & $\begin{array}{l}\text { C. coli } \\
\text { (6) }\end{array}$ & $\begin{array}{l}\text { C. jejuni } \\
\text { (32) }\end{array}$ & $\begin{array}{l}\text { C. coli } \\
\text { (8) }\end{array}$ & & \\
\hline 0.45 & 10 & 7 & - & $\begin{array}{c}1 \\
(1.92)\end{array}$ & - & - & - & - & $\begin{array}{c}1 \\
(0.40)\end{array}$ & \multirow{10}{*}{ MDR } \\
\hline 0.5 & 11 & 8 & - & $\begin{array}{c}1 \\
(1.92)\end{array}$ & - & - & - & - & $\begin{array}{c}1 \\
(0.40)\end{array}$ & \\
\hline 0.55 & 12 & 7 & $\begin{array}{c}1 \\
(0.86) \\
\end{array}$ & $\begin{array}{c}1 \\
(1.92) \\
\end{array}$ & - & - & - & - & $\begin{array}{c}2 \\
(0.81) \\
\end{array}$ & \\
\hline 0.59 & 13 & 8 & $\begin{array}{c}4 \\
(3.44)\end{array}$ & $\begin{array}{c}2 \\
(3.85)\end{array}$ & - & - & $\begin{array}{c}4 \\
(12.5)\end{array}$ & - & $\begin{array}{c}10 \\
(4.05)\end{array}$ & \\
\hline 0.64 & 14 & $7,8,9,10$ & $\begin{array}{c}5 \\
(4.31) \\
\end{array}$ & $\begin{array}{c}3 \\
(5.77) \\
\end{array}$ & $\begin{array}{c}5 \\
(15.15)\end{array}$ & - & $\begin{array}{c}3 \\
(9.38)\end{array}$ & - & $\begin{array}{c}16 \\
(6.48)\end{array}$ & \\
\hline 0.68 & 15 & $7,8,9$ & $\begin{array}{c}18 \\
(15.52)\end{array}$ & $\begin{array}{c}1 \\
(1.92)\end{array}$ & $\begin{array}{c}4 \\
(12.12)\end{array}$ & $\begin{array}{c}2 \\
(33.33)\end{array}$ & $\begin{array}{c}1 \\
(3.13)\end{array}$ & - & $\begin{array}{c}26 \\
(10.53)\end{array}$ & \\
\hline 0.73 & 16 & $7,8,9,10$ & $\begin{array}{c}11 \\
(50)\end{array}$ & $\begin{array}{c}3 \\
(5.77)\end{array}$ & $\begin{array}{c}7 \\
(21.21)\end{array}$ & $\begin{array}{c}2 \\
(33.33)\end{array}$ & $\begin{array}{c}6 \\
(18.75)\end{array}$ & $3(37.5)$ & $\begin{array}{c}32 \\
(12.96)\end{array}$ & \\
\hline 0.77 & 17 & $7,8,9,10$ & $\begin{array}{c}22 \\
(18.97)\end{array}$ & $\begin{array}{c}17 \\
(32.69)\end{array}$ & $\begin{array}{c}10 \\
(30.30)\end{array}$ & - & $\begin{array}{c}4 \\
(12.5)\end{array}$ & $\begin{array}{c}2 \\
(25)\end{array}$ & $\begin{array}{c}55 \\
(22.27)\end{array}$ & \\
\hline 0.82 & 18 & $8,9,10$ & $\begin{array}{c}15 \\
(12.93)\end{array}$ & $\begin{array}{c}12 \\
(23.08)\end{array}$ & $\begin{array}{c}5 \\
(15.15)\end{array}$ & $2(33.33)$ & $5(15.63)$ & - & $\begin{array}{c}39 \\
(15.79)\end{array}$ & \\
\hline 0.86 & 19 & $7,8,9,10$ & $\begin{array}{c}19 \\
(16.32)\end{array}$ & $\begin{array}{c}4 \\
(7.69)\end{array}$ & - & - & $\begin{array}{c}1 \\
(3.13)\end{array}$ & $\begin{array}{c}1 \\
(12.5)\end{array}$ & $\begin{array}{c}25 \\
(10.12)\end{array}$ & \\
\hline 0.91 & 20 & 9,10 & $\begin{array}{c}10 \\
(8.62)\end{array}$ & $\begin{array}{c}5 \\
(9.62)\end{array}$ & - & - & $6(18.75)$ & $\begin{array}{c}2 \\
(25)\end{array}$ & $\begin{array}{c}23 \\
(9.31)\end{array}$ & \multirow{2}{*}{ XDR } \\
\hline 0.95 & 21 & 9,10 & $\begin{array}{c}9 \\
(7.76)\end{array}$ & $\begin{array}{c}2 \\
(3.85)\end{array}$ & $\begin{array}{c}2 \\
(6.06)\end{array}$ & - & $\begin{array}{c}2 \\
(6.25)\end{array}$ & - & $\begin{array}{c}15 \\
(6.07)\end{array}$ & \\
\hline 1 & 22 & 10 & $\begin{array}{c}2 \\
(1.72)\end{array}$ & - & - & - & - & - & $\begin{array}{c}2 \\
(0.81)\end{array}$ & PDR \\
\hline
\end{tabular}

AMC*: antimicrobial classes, MDR: multidrug resistant, XDR: extensively drug resistant, PDR: pan drug resistant

\section{DISCUSSION}

Human campylobacteriosis is caused primarily due to consuming chicken meat, raw milk and inadequately pasteurized milk. The presence of campylobacter pathogens in food animals is particularly worrying for human health and controlling them has a significant implication on health of the public. The current study includes more completed and updated information about the frequency and the antimicrobial resistance of $C$. jejuni and $C$. coli from different sources in Zagazig.
Our results demonstrated that the occurrence of Campylobacter spp. in samples obtained from broilers was $86.15 \%$ (168 campylobacter isolates out of 195 samples). These results are consistent with previous reports in Poland $(87.2 \%)$ (Wieczorek $\boldsymbol{e t}$ al., 2012) and Algeria (85\%) (Messad $\boldsymbol{e t}$ al., 2014). Moreover, higher prevalence rates of Campylobacter spp. were reported in Italy (100\%) (Giacomelli et al., 2014) and Algeria (96\%) (Guessoum et al., 2016). On the other hand, low prevalence rates of avian Campylobacter spp. were previously documented in Harare $(60.2 \%)$ (Simango, 2013) and India (44.9\%) (Vaishnavi, 2015). In Egypt, in Giza and Cairo Governorates, 7 out of 360 chicken cloacal 
swabs collected from different households, farms and shops were positive for Campylobacter spp. (1.9\%) (Ghoneim et al., 2020). In Assiut Governorate, 24\% of 104 chicken carcasses from two slaughterhouses contained Campylobacter spp. (Abushahba et al., 2018).

In regard to Campylobacter spp. distribution, $C$. jejuni was the most common isolated distribution is comparable to other previous reports in Ireland, $68.9 \%$ and $32.4 \%$ and Austria, $65.1 \%$ and $33.3 \%$ for $C$. jejuni and C. coli, respectively (EFSA, 2010). A recent studies also documented that $C$. jejuni was more often isolated from chicken in Tunisia (Gharbi $\boldsymbol{e t}$ al., 2018) and Egypt (Ghoneim $\boldsymbol{e}$ al., 2020). However, another study conducted in Italy reported that $C$. coli was the prevailing Campylobacter spp. among the isolates of chicken origin (Nobile et al., 2013). Generally, the variations in Campylobacter spp. isolation rates between different studies could be attributed to different reasons such as the type of examined samples, location, climate factors, hygienic measures and isolation as well as techniques of identification (Jorgensen et al., 2011; Chatur $\boldsymbol{e t}$ al., 2014)

Herein, Campylobacter spp. was isolated from $82.98 \%$ of raw milk samples, which is higher than levels obtained by El-Kholy et al., (13\%) (El-Kholy $\boldsymbol{e t}$ al., 2016) and El-Zamkan and Hameed (22\%) (El-Zamkan and Hameed, 2016) in Egypt, Kashoma et al., (13.4\%) in Tanzania (Kashoma et al., 2016) and Andrzejewska et al., (11.8\%) in Poland (Andrzejewska et al., 2019). C. jejun were identified in the current study from $70.21 \%$ of the examined raw milk samples. However results obtained by Hussain et al. in Pakistan, showed that the incidence rate of $C$. jejuni was $92.4 \%$ (Hussain et al., 2007).

The more frequent second source of campylobacteriosis is raw milk (Kashoma $\boldsymbol{e}$ al., 2016). Popularly, consuming organic and raw food has been increased, so consumers need to be aware of the danger related to consumption of unpasteurized milk (Mie et al., 2017). The high occurrence of Campylobacter spp. in raw milk in the current study could be due to environmental contamination of milk during or after milking with infected animal wastes or from contaminated external surface of the teats, unsanitary equipment or hands of workers and storage practices (Saad et al., 2007).

The occurrence of Campylobacter spp. in human stool samples was $90.91 \%$. Thi result was higher than those reported Giza (16.7\%) (Hassanain, 2011) and Assuit Governates (27.5\%) (Abushahba et al., 2018) in Egypt. Also higher than in other countries; Ethiopia (72.7\%) (Ewnetu and Mihret, 2010), Nigeria (62.7\%) (Gwimi et al., 2015), Ghana (20.3\%) (Karikari et al., 2017) and Poland $(9.6 \%$ (Szczepanska et al., 2017). C. jejuni were identified in the current study from $72.73 \%$ of the examined human stool samples. This result were nearly similar to 69.3\% and 76.9\% reported in Romania (Sorokin et al., 2007) and Egypt (Abd E Tawab et al., 2018), respectively. The significantly higher percentage of Campylobacter spp. from human stool samples in our study could be ascribed to incorporation of stool samples mainly obtained from individuals with diarrhea rather than inspection of campylobacteriosis in population in general.

In the last few years, antimicrobial resistance in foodborne microorganisms involving campylobacters is considered one of the vitally important problems on public health (Wieczorek and Osek, 2013; EFSA, 2015). In clinical setting, macrolides (i.e erythromycin), fluoroquinolones (i.e ciprofloxacin) and tetracycline are widely used in the treatment of campylobacter infections because of their availability and low cost.

Some reports have demonstrated a slow increase in the resistance rate of campylobacters to macrolides, which are considered the first drugs of choice for treatment of campylobacteriosis, especially in paediatric patients (Kurinčič et al. 2007; Wieczorek and Osek, 2013). In this study, all campylobacter isolates from chicken, human and cattle were resistant to erythromycin (100\% each). This is in accordance with a recent report in Egypt, where 100\% of campylobacter isolates from chicken were resistant to erythromycin (Abd El Tawab et al., 2018). On the other hand, lower resistance rates to this drug were recorded for campylobacter isolates from milk samples in Iran (7.69\%) (Rahimi et al., 2013) and from human in China (21.8\%) (Pan $\boldsymbol{e t}$ al., 2016). Ladely et al. reported that exposing campylobacters to macrolides for a long duration results in their resistance (Ladely et al., 2007).

Our research demonstrated a high level of ciprofloxacin resistance among campylobacter isolates from chicken, human and cattle $(75 \%, 75 \%$ and $61.54 \%$, respectively). Similarly, a higher resistance rate $(99.2 \%)$ was also reported for campylobacter isolates from chicken in Tunisia (Gharbi et al., 2018). Using quinolones in veterinary fields leads to the emergency of ciprofloxacin resistance among campylobacters isolated from human and chicken (Engberg et al., 2004; Gupta et al., 2004). However, small number of campylobacter isolates was resistant to quinolones in some countries such as Australia, in which quinolones were not allowed to be used in chicken production (Skarp et al., 2016). Due to the increased resistance to fluoroquinolones and quinolones, these agents became ineffective in campylobacteriosis treatment (Han et al., 2016).

There were also high resistance rates to doxycycline in our campylobacter isolates from human, chicken and cattle $(90 \%, 82.14 \%$ and $69.23 \%$ respectively). This is in accordance with a previous report carried out on campylobacter isolates from raw milk (71.43 \%) in Poland (Wysok et al., 2011). Due to the broad spectrum of activity and the low cost of tetracyclines, they have been widely used in the prophylaxis therapy of human and animal infections and as feed complements for chicken. These have resulted in the emergence of high resistant bacteria (Hassanain, 2011). Alarmingly, the increased resistance of campylobacter isolates to antimicrobials, particularly erythromycin, (fluoro) quinolones and tetracycline can result in failure in the treatment resulting in higher illness and death rates in humans (Zhu et al., 2006).

The high resistance of campylobacter isolates to the antimicrobials in the present study might be due to the widespread and the uncontrolled use of these agents in veterinary medicine as growth promoters or in human and animal treatments. This gives a reflection about the extent of using these antimicrobials in Egypt and therefore proposes a challenge to the management of campylobacter infections. Nowadays, the emergence of MDR campylobacter isolates is becoming a growing challenge as it can impair the effective therapy of campylobacter infections. In the current study, there were XDR and PDR campylobacter isolates especially to the antimicrobials those are used in the treatment of campylobacter infections leading to more difficulties in controlling these infections. It is interesting to note that all Campylobacter spp. in this study had an MAR index greater than 0.45, which indicates a high frequency of antibiotics usage in Egypt This worrisome resistance rates were also recorded for campylobacter isolates from chicken in many countries like Italy (100\%) (Fraqueza et al., 2014), Algeria (100\%) (Messad et al., 2014) and Pakistan (90.4\%) (Nisar et al., 2017) The appearance of MDR can be attributed to the attainment of several resistance determinants in the same DNA molecule or individual determinants such as multiple drug pumps (Levy, 2002). The CmeABC (multi-drug efflux pump) has been concerned with the campylobacter resistance mechanisms to macrolides, fluoroquinolones and tetracyclines (Ventola, 2015).

In order to confirm the identification and discrimination of C. jejuni and C. coli conventional PCR has been used targeting mapA and ceuE genes, respectively (Ghoneim et al., 2020). In the present work the conventional PCR results confirmed the identification of thirty eight campylobacter isolates (29 and 9 phenotypically suspected $C$. jejuni and $C$. coli isolates, respectively). Accordingly, the conventional culture methods and biochemical reactions were $100 \%$ in accordance with the results of PCR for identification and differentiation of $C$. jejuni and C. coli. The same results were reported in Egypt (Girgis et al., 2014).

This study is limited by some factors, mainly related to lack of fund: first, the dependence on PCR for characterizing the bacterial species could be subjected to lack of specificity, and other techniques (e.g. MALDI-TOF) is recommended and will be taken into consideration in future studies. However, including control positive and negative compensated, to some extent, this limitation. Other shortcoming is that we depended on phenotypic approach to define the resistance to antimicrobials. Indeed, an ongoing project that involves this point on the same isolates is in plane for future publication. In addition, we were not able to include other sample types from "Cattle" (i.e. other than milk) due to unavailability and lack of access to the sampled animals.

\section{CONCLUSION}

Our data demonstrated that the relatively high isolation rate of campylobacters from chicken, raw milk and human stool samples in addition to the development of MDR strains to multiple antimicrobial classes, especially to macrolides, quinolones and tetracycline are alarming situations with potential serious consequences to the health of human. Therefore, there is a need to reduce using antimicrobial agents in food animals and to implement specific contro procedures to decrease contamination levels by campylobacters to prevent resistant campylobacter strains from emerging and spreading.

\section{REFERENCES}

Abd El Tawab, A. A., Ammar, A. A., Ahmed, H. A. \& Hefny, A. A. (2018), Bacteriological and Molecular Identification of some Campylobacter Species in Broilers and their Macrolide Resistance Profile. Benha Veterinary Medical Journal, 34(1), 374-391. https://dx.doi.org/10.21608/bvmj.2018.54483

Abulreesh, H. H., Organji, S. R., Elbanna, K., Osman, G. E. H., Almalki, M. H K. \& Ahmad, I. (2017). Campylobacter in the environment: A major threat to public health. Asian Pacific Journal of Tropical Disease, 7(6), 374-384. https://doi.org/10.12980/apitd.7.2017d6-392

Abushahba, M. F., Ahmed, S. O., Ibrahim, A. A. \& Mosa, H. A. (2018) Prevalence of zoonotic species of Campylobacter in broiler chicken and humans in Assiut governorate, Egypt. Approaches Poult. Dairy Vet. Sci, 3(4), 1-9. https:// dx.doi.org/10.31031/apdv.2018.03.000568

Andrzejewska, M., Szczepańska, B., Śpica, D. \& Klawe, J. J. (2019). Prevalence, Virulence, and Antimicrobial Resistance of Campylobacter spp. in Raw Milk Beef, and Pork Meat in Northern Poland. Foods, 8(9), 420. https:// dx.doi.org/10.3390/foods8090420

Awadallah, M. A. I., Ahmed, H. A., El-Gedawy, A. A., \& Saad, A. M. (2014) Molecular identification of $C$. jejuni and $C$. coli in chicken and humans, at Zagazig, Egypt, with reference to the survival of $C$. jejuni in chicken meat at refrigeration and freezing temperatures. International Food Research Journal, 21(5), 1801. 
Bauer, A. W., Kirby, W. M. M., Sherris, J. C. \& Turck, M. (1966). Antibiotic susceptibility testing by a standardized single disk method. American journal of clinical pathology, 45(4), 493-496. https://dx.doi.org/10.1093/ajcp/45.4 ts.493 Bouhamed, R., Bouayad, L., Messad, S., Zenia, S., Naïm, M. \& Hamdi, T.-M (2018). Sources of contamination, prevalence, and antimicrobial resistance of thermophilic Campylobacter isolated from turkeys. Veterinary World, 11(8), 1074. https:// dx.doi.org/10.14202/vetworld.2018.1074-1081

Chatur, Y. A., Brahmbhatt, M. N., Modi, S., \& Nayak, J. B. (2014). Fluoroquinolone resistance and detection of topoisomerase gene mutation in Campylobacter jejuni isolated from animal and human sources. Int. J. Curr. Microbiol. App. Sci, 3(6), 773-783.

CLSI, (2013). Performance Standards for Antimicrobial Susceptibility Testing, Twenty-Third Informational Supplement. CLSI document M100-S23. Wayne, PA. Clinical and Laboratory Standards Institute CLSI, (2016). Performance Standards for Antimicrobial Susceptibility Testing 26th ed. CLSI supplement M100S. Wayne, PA. Clinical and Laboratory Standards Institute.

Eberle, K. N.\& Kiess, A. S. (2012). Phenotypic and genotypic methods for typing Campylobacter jejuni and Campylobacter coli in poultry. Poultry science, 91(1), 255-264. https://dx.doi.org/10.3382/ps.2011-01414

European Food Safety Authority (EFSA)(2010). 'The community summary report on trends and sources of zoonoses'. Zoonotic agents and foodborne outbreaks in the European Union (2008). EFSA Journal, 8(3):1496-1906.

European Food Safety Authority, \& European Centre for Disease Prevention and Control. (2015). EU Summary Report on antimicrobial resistance in zoonotic an indicator bacteria from humans, animals and food in 2013. EFSA Journal, 13(2), 4036.

El-Hamid, M. I., El-Aziz, A., Khairy, N., Samir, M., Abo Remela, E. M., ElNaenaeey, E. S. Y., ... \& Mosbah, R. A. (2019). Genetic Diversity of Campylobacter jejuni Isolated From Avian and Human Sources in $\begin{array}{lll}\text { Egypt. Frontiers in } & \text { microbiology, 10, }\end{array}$ https://dx.doi.org/10.3389/fmicb.2019.02353

El-Kholy, A. M., Meshref, A. M. S., El-Gedawy, A. A. \& Esam, R. M. (2016) Prevalence of Campylobacter species in milk and some dairy products. Journa of Veterinary Medical Research, 23(2), 133-142. https://dx.doi.org/10.21608/jvmr.2016.43234

El-Tras, W. F., Holt, H. R., Tayel, A. A. \& El-Kady, N. N. (2015) Campylobacter infections in children exposed to infected backyard poultry in Egypt. Epidemiology \& Infection, 143(2), 308-315. https://dx.doi.org/10.1017/s095026881400096x

El-Zamkan, M. A. \& Abdel Hameed, K. G. (2016). Prevalence of Campylobacter jejuni and Campylobacter coli in raw milk and some dairy products. Veterinary World, 9(10), 1147-1151. https://dx.doi.org/10.14202/vetworld.2016.1147-1151 Engberg, J., Neimann, J., Nielsen, E. M., Aarestrup, F. M. \& Fussing, V. (2004) Quinolone-resistant Campylobacter infections: risk factors and clinica consequences. Emerging infectious diseases, 10(6), 1056-1063. https:// dx.doi.org/10.3201/eid1006.030669

European Committee on Antimicrobial Susceptibility Testing. (2017). Breakpoin tables for interpretation of MICs and zone diameters. Version 7.1, valid from 2017-03-10. URL: http://www.

org/fileadmin/src/media/PDFs/EUCAST_files/Breakpoint__tables/v_7. 1 Breakpoint Tables. pdf (12.11. 2017)

Ewnetu, D. \& Mihret, A. (2010). Prevalence and antimicrobial resistance of Campylobacter isolates from humans and chickens in Bahir Dar, Ethiopia. Foodborne pathogens and disease, 7(6), 667-670. https://dx.doi.org/10.1089/fpd.2009.0433

Fraqueza, M. J., Martins, A., Borges, A. C., Fernandes, M. H., Fernandes, M. J. Vaz, Y., ...\& Barreto, A. S. (2014). Antimicrobial resistance among Campylobacter spp. strains isolated from different poultry production systems at slaughterhouse level. Poultry science, 93(6), 1578-1586. https://dx.doi.org/10.3382/ps.2013-03729

García-Sánchez, L., Melero, B., \& Rovira, J. (2018). Campylobacter in the food chain. In Advances in Food and Nutrition Research (Vol. 86, pp. 215-252). Academic Press. https://dx.doi.org/10.1016/bs.afnr.2018.04.005

Gharbi, M., Béjaoui, A., Ben Hamda, C., Jouini, A., Ghedira, K., Zrelli, C., ...\& Maaroufi, A (2018). Prevalence and antibiotic resistance patterns of Campylobacter spp. isolated from broiler chickens in the north of Tunisia. BioMed research international, 2018,1-7. https://dx.doi.org/10.1155/2018/7943786

GHONEIM, N. H., ABDEL-MOEIN, K. A.-A., BARAKAT, A. M. A. K., HEGAZI, A. G., ABD EL-RAZIK, K. A. E.-H. \& SADEK, S. A. S. (2020) Isolation and molecular characterization of Campylobacter jejuni from chicken and human stool samples in Egypt. Food Science and Technology. https://dx.doi.org/10.1590/fst.01620

Ghoneim, N. H., Sabry, M. A., Ahmed, Z. S. \& Elshafiee, E. A. (2020). Campylobacter Species Isolated from Chickens in Egypt: Molecular Epidemiology and Antimicrobial Resistance. Pakistan Journal of Zoology, 52 917.https:// dx.doi.org/10.17582/journal.piz/20190324080346

Giacomelli, M., Salata, C., Martini, M., Montesissa, C., \& Piccirillo, A. (2014) Antimicrobial resistance of Campylobacter jejuni and Campylobacter coli from poultry in Italy. Microbial Drug Resistance, 20(2), 181-188.https:// dx.doi.org/10.1089/mdr.2013.0110

Girgis, S. A., Rashad, S. S., Othman, H. B., Bassim, H. H., Kassem, N. N., \& ElSayed, F. M. (2014). Multiplex PCR for identification and differentiation of Campylobacter species and their antimicrobial susceptibility pattern in Egyptian patients. Int J Curr Microbiol App Sci, 3(4), 861-75.

Guessoum, M., Guechi, Z., Aigoun, F., Mahrane, S. \& Hachemi, A. (2016). Campylobacter in sheep, calves and broiler chickens in the central region of Algeria: Phenotypic and antimicrobial resistance profiles. Afr J Microbiol Res, 10(39), 1662-1667. https://dx.doi.org/10.5897/ajmr2016.8238

Gupta, A., Nelson, J. M., Barrett, T. J., Tauxe, R. V., Rossiter, S. P., .. \&Friedman, C. R. (2004). Antimicrobial resistance among campylobacter strains, United States, 1997-2001. Emerging infectious diseases, 10(6), 1102-1109. https:// dx.doi.org/10.3201/eid1006.030635

Gwimi, P. B., Faleke, O. O., Salihu, M. D., Magaji, A. A., Abubakar, M. B., Nwankwo, I. O., \& Ibitoye, E. B. (2015). Prevalence of Campylobacter species in fecal samples of pigs and humans from Zuru Kebbi State, Nigeria. International Journal of One Health, 1, 1-5. https:/dx.doi.org/10.14202/ijoh.2015.1-5

Han, X., Zhu, D., Lai, H., Zeng, H., Zhou, K., Zou, L., ... Liu, S. (2016). Prevalence, antimicrobial resistance profiling and genetic diversity of Campylobacter jejuni and Campylobacter coli isolated from broilers at slaughter in China. Food Control, 69, 160-170. https://dx.doi.org/10.1016/j.foodcont.2016.04.051

Hassanain, N. A. (2011). Antimicrobial resistant Campylobacter jejuni isolated from humans and animals in Egypt. Global Veterinaria, 6(2), 195-200.

Humphrey, T., O'Brien, S. \& Madsen, M. (2007). Campylobacters as zoonotic pathogens: a food production perspective. International journal of food microbiology, $117(3)$,

237-257.

https://dx.doi.org/10.1016/j.ijfoodmicro.2007.01.006

Hussain, I., Mahmood, M. S., Akhtar, M. \& Khan, A. (2007). Prevalence of Campylobacter species in meat, milk and other food commodities in Pakistan Food microbiology, 24(3), 219-222. https://dx.doi.org/10.1016/j.fm.2006.06.001 ISO (International Organisation for Standardisation). (2006). ISO 10272-1: 2006 Microbiology of food and animal feeding stuffs-Horizontal method for detection and enumeration of Campylobacter spp.-Part 1: Detection method. Part, 1.

Jorgensen, F., Ellis-Iversen, J., Rushton, S., Bull, S., Harris, S., Bryan, S. Gonzalez, A. \& Humphrey, T. (2011). Influence of season and geography on Campylobacter jejuni and $C$. coli subtypes in housed broiler flocks reared in Great Britain. Applied and environmental microbiology, 77(11), 3741-3748. https://dx.doi.org/10.1128/aem.02444-10

Karikari, A. B., Obiri-Danso, K., Frimpong, E. H. \& Krogfelt, K. A. (2017) Antibiotic resistance in Campylobacter isolated from patients with gastroenteritis in a teaching hospital in Ghana. Open Journal of Medical Microbiology, 7(1), 111. https:// dx.doi.org/10.4236/ojmm.2017.71001

Kashoma, I. P., Kassem, I. I., John, J., Kessy, B. M., Gebreyes, W., Kazwala, R R. \& Rajashekara, G. (2016). Prevalence and antimicrobial resistance of campylobacter isolated from dressed beef carcasses and raw milk in Tanzania. $\begin{array}{llll}\text { Microbial drug } & \text { resistance, } & 22(1), & 40-52\end{array}$ https://dx.doi.org/10.1089/mdr.2015.0079

Krumperman, P. H. (1983). Multiple antibiotic resistance indexing of Escherichia coli to identify high-risk sources of fecal contamination of foods Appl. Environ. $\quad$ Microbiol., $46 \quad$ (1), $165-170$. https://doi.org/10.1128/aem.46.1.165-170.1983

Kurinčič, M., Botteldoorn, N., Herman, L. \& Smole Možina, S. (2007) Mechanisms of erythromycin resistance of Campylobacter spp. isolated from food, animals and humans. International journal of food microbiology, 120(1-2), 186-190. https://dx.doi.org/10.1016/j.ijfoodmicro.2007.03.012

Ladely, S. R., Harrison, M. A., Fedorka-Cray, P. J., Berrang, M. E., Englen, M. D. \& Meinersmann, R. J. (2007). Development of macrolide-resistant Campylobacter in broilers administered subtherapeutic or therapeutic concentrations of tylosin. Journal of food protection, 70(8), 1945-1951. https://dx.doi.org/10.4315/0362-028x-70.8.1945

Levy, S. B. (2002). Factors impacting on the problem of antibiotic resistance. Journal of Antimicrobial Chemotherapy, 49(1), 25-30. https://dx.doi.org/10.1093/jac/49.1.25

Magiorakos, A. P., Srinivasan, A., Carey, R. T., Carmeli, Y., Falagas, M. T. Giske, C. T., ... \& Paterson, D. T. (2012). Multidrug-resistant, extensively drugresistant and pandrug-resistant bacteria: an international expert proposal for interim standard definitions for acquired resistance. Clinical microbiology and infection, 18(3), 268-281.https://dx.doi.org/10.1111/j.1469-0691.2011.03570.x Messad, S., Hamdi, T.-M., Bouhamed, R., Ramdani-Bouguessa, N. \& Tazir, M (2014). Frequency of contamination and antimicrobial resistance of thermotolerant Campylobacter isolated from some broiler farms and slaughterhouses in the region of Algiers. Food control, 40, 324-328. https://dx.doi.org/10.1016/j.foodcont.2013.12.016

Mie, A., Andersen, H. R., Gunnarsson, S., Kahl, J., Kesse-Guyot, E., Rembiałkowska, E., ... \& Grandjean, P. (2017). Human health implications of organic food and organic agriculture: a comprehensive review. Environmental Health, 16 (1). https:// dx.doi.org/10.1186/s12940-017-0315-4 
Narvaez-Bravo, C., Taboada, E. N., Mutschall, S. K. \& Aslam, M. (2017). Epidemiology of antimicrobial resistant Campylobacter spp. isolated from retai meats in Canada. International journal of food microbiology, 253, 43-47. https:// dx.doi.org/10.1016/j.ijfoodmicro.2017.04.019

Nisar, M., Mushtaq, M. H., Shehzad, W., Hussain, A., Muhammad, J., Nagaraja, K. V. \& Goyal, S. M. (2017). Prevalence and antimicrobial resistance patterns of Campylobacter spp. isolated from retail meat in Lahore, Pakistan. Food Control, 80, 327-332. https://dx.doi.org/10.1016/j.foodcont.2017.03.048

Nobile, C. G., Costantino, R., Bianco, A., Pileggi, C. \& Pavia, M. (2013) Prevalence and pattern of antibiotic resistance of Campylobacter spp. in poultry meat in Southern Italy. Food Control, 32(2), 715-718. https:// dx.doi.org/10.1016/j.foodcont.2013.02.011

Pan, H., Ge, Y., Xu, H., Zhang, J., Kuang, D., Yang, X., ...\& Meng, J. (2016) Molecular characterization, antimicrobial resistance and Caco-2 cell invasion potential of Campylobacter jejuni/coli from young children with diarrhea. The Pediatric infectious disease journal, 35(3), 330-334. https://dx.doi.org/10.1097/inf.0000000000001016

Quinn, P. J., Carter, M. E., Markey, B., \& Carter, G. R. (1994). Campylobacter species. Clinical Veterinary Microbiology, 268-272.

Rahimi, E., Sepehri, S., \& Momtaz, H. (2013). Prevalence of Campylobacter species in milk and dairy products in Iran. REVUE DE MEDECINE VETERINAIRE, 164(5), 283-288.

Saad, N., Ahmed, A., Haleem, A. A., \& Nassife, T. (2007). Incidence of Campylobacter species in milk and some milk products. Assiut Vet. Med. $J, 33114,106-122$

Saeed, A., Abd, H. \& Sandstrom, G. (2015). Microbial aetiology of acute diarrhoea in children under five years of age in Khartoum, Sudan. Journal of medical microbiology, 64(4), 432-437. https://dx.doi.org/10.1099/jmm.0.000043 Sahin, O., Morishita, T. Y. \& Zhang, Q. (2002). Campylobacter colonization in poultry: sources of infection and modes of transmission. Animal Health Research Reviews, 3(2), 95-105. https://dx.doi.org/10.1079/ahrr200244

Sainato, R., ElGendy, A., Poly, F., Kuroiwa, J., Guerry, P., Riddle, M. S. \& Porter, C. K. (2018). Epidemiology of Campylobacter infections among children in Egypt. The American journal of tropical medicine and hygiene, 98(2), 581585. https://dx.doi.org/10.4269/ajtmh.17-0469

Shin, E. J., \& Lee, Y. H. (2009). Comparison of three different methods for Campylobacter isolation from porcine intestines. Journal of microbiology and biotechnology, 19(7), 647-650. https://dx.doi.org/10.4014/jmb.0807.432

Silva, J., Leite, D., Fernandes, M., Mena, C., Gibbs, P. A., \& Teixeira, P. (2011) Campylobacter spp. as a Foodborne Pathogen: A Review. Frontiers in microbiology, 2, 200. https://dx.doi.org/10.3389/fmicb.2011.00200

Simango, C. (2013). Antimicrobial susceptibility of Campylobacter species. Southern African Journal of Epidemiology and Infection, 28(3), 139-142. https://dx.doi.org/10.1080/10158782.2013.11441535

Skarp, C., Hänninen, M.-L. \& Rautelin, H. (2016). Campylobacteriosis: the role of poultry meat. Clinical Microbiology and Infection, 22(2), 103-109. https://dx.doi.org/10.1016/j.cmi.2015.11.019

Sorokin, M., Usein, C. R., Irimia, M., \& Damian, M. (2007). A laboratory-based survey of Campylobacter infections in Prahova County. Roum Arch Microbiol Immunol, 66(3-4), 85-9.

Szczepanska, B., Andrzejewska, M., Spica, D. \& Klawe, J. J. (2017). Prevalence and antimicrobial resistance of Campylobacter jejuni and Campylobacter coli isolated from children and environmental sources in urban and suburban areas. BMC microbiology, 17 (1). https://dx.doi.org/10.1186/s12866-017-0991-9

Thomrongsuwannakij, T., Blackall, P. J. \& Chansiripornchai, N. (2017). A study on Campylobacter jejuni and Campylobacter coli through commercial broiler production chains in Thailand: antimicrobial resistance, the characterization of DNA gyrase subunit A mutation, and genetic diversity by flagellin A gene restriction fragment length polymorphism. Avian diseases, 61(2), 186-197. https://dx.doi.org/10.1637/11546-120116-reg.1

Vaishnavi, C. (2015). Isolation of Campylobacters from intestinal tract of Poultry in Northern Region of India. Advances in Microbiology, 5(12), 797-806. https://dx.doi.org/10.4236/aim.2015.512084

Ventola, C. L. (2015). The antibiotic resistance crisis: part 1: causes and threats. Pharmacy and therapeutics, 40(4), 277.

Wang, G., Clark, C. G., Taylor, T. M., Pucknell, C., Barton, C., Price, L., ... \& Rodgers, F. G. (2002). Colony multiplex PCR assay for identification and differentiation of Campylobacter jejuni, C. coli, C. lari, C. upsaliensis, and $C$. fetus subsp. fetus. Journal of Clinical Microbiology, 40(12), 4744-4747. https://dx.doi.org/10.1128/jcm.40.12.4744-4747.2002

WHO. (2015). The World Health Organization Estimates of the Global Burden of Foodborne Dieseases: FERG Project Report. http://www.who.int/foodsafety/areas_work/foodborne-diseases/ferg/en/ Wieczorek, K. \& Osek, J. (2013). Antimicrobial resistance mechanisms among Campylobacter. BioMed research international, 2013,1-12. https://dx.doi.org/10.1155/2013/340605

Wieczorek, K., Szewczyk, R. \& Osek, J. (2012). Prevalence, antimicrobial resistance, and molecular characterization of Campylobacter jejuni and C. col isolated from retail raw meat in Poland. Veterinarni Medicina, 57(6). https:// dx.doi.org/10.17221/6016-vetmed
Wysok, B., Wiszniewska-Łaszczych, A., Uradziński, J. \& Szteyn, J. (2011). Prevalence and antimicrobial resistance of Campylobacter in raw milk in the selected areas of Poland. Polish journal of veterinary sciences, 14 (3). https:// dx.doi.org/10.2478/v10181-011-0070-3

Zhou, J., Zhang, M., Yang, W., Fang, Y., Wang, G. \& Hou, F. (2016). A seventeen-year observation of the antimicrobial susceptibility of clinical Campylobacter jejuni and the molecular mechanisms of erythromycin-resistan isolates in Beijing, China. International Journal of Infectious Diseases, 42, 28 33. https://dx.doi.org/10.1016/j.ijid.2015.11.005

Zhu, J., Zhang, Y., Hua, X., Hou, J. \& Jiang, Y. (2006). Antibiotic resistance in Campylobacter. Reviews in Medical Microbiology, 17(4), 107-112. https://dx.doi.org/10.1097/mrm.0b013e3280c4d106 